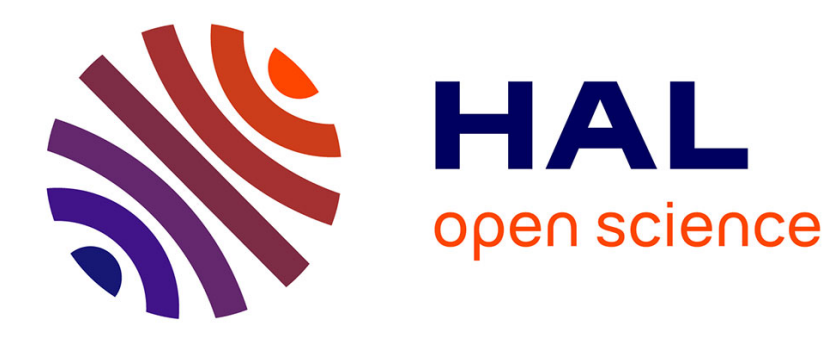

\title{
On the Minimum Size of a Contraction-Universal Tree
} Olivier Bodini

\section{To cite this version:}

Olivier Bodini. On the Minimum Size of a Contraction-Universal Tree. 2003. hal-00432221

\section{HAL Id: hal-00432221 \\ https://hal.science/hal-00432221}

Preprint submitted on 14 Nov 2009

HAL is a multi-disciplinary open access archive for the deposit and dissemination of scientific research documents, whether they are published or not. The documents may come from teaching and research institutions in France or abroad, or from public or private research centers.
L'archive ouverte pluridisciplinaire $\mathbf{H A L}$, est destinée au dépôt et à la diffusion de documents scientifiques de niveau recherche, publiés ou non, émanant des établissements d'enseignement et de recherche français ou étrangers, des laboratoires publics ou privés. 


\title{
On the Minimum Size of a Contraction-Universal Tree
}

\author{
Olivier Bodini \\ LIP, École Normale Supérieure de Lyon, 46 Allée d'Italie, 69364 Lyon Cedex 05, \\ France.
}

\begin{abstract}
A tree $T_{u n i}$ is $m$-universal for the class of trees if for every tree $T$ of size $m, T$ can be obtained from $T_{u n i}$ by successive contractions of edges. We prove that a $m$-universal tree for the class of trees has at least $m \ln (m)+(\gamma-1) m+O(1)$ edges where $\gamma$ is the Euler's constant and we build such a tree with less than $m^{c}$ edges for a fixed constant $c=1.984 \ldots$
\end{abstract}

\section{Introduction}

What is the minimum size of an object in which every object of size $m$ embeds? Issued from the category theory, questions of this kind appeared in graph theory. For instance, R. Rado [1] proved the existence of an "initial countable graph". Recently, Z. Füredi and P. Komjàth [2] studied a connected question.

We use here the following definition : given a sub-class $C$ of graphs (trees, planar graphs, etc.), a graph $G_{u n i}$ is $m$-universal for $C$ if for every graph $G$ of size $m$ in $C, G$ is a minor of $G_{u n i}$, i.e. it can be obtained from $G_{u n i}$ by successive contractions or deletions of edges.

Inspired by the Robertson and Seymour work [3] on graph minors, P. Duchet asked whether a polynomial bound in $m$ could be found for the size of a $m$ universal tree for the class of trees. We give here a positive sub-quadratic answer.

From an applied point of view, such an object would possibly allows us to define a tree from the representation of its contraction.

The main results of this paper are the following theorems which give bounds for the minimum size of a $m$-universal tree for the class of trees :

Theorem 1. A m-universal tree for the class of trees has at least $m \ln (m)+$ $(\gamma-1) m+O(1)$ edges where $\gamma$ is the Euler's constant.

Theorem 2. There exists a $m$-universal tree $T_{\text {uni }}$ for the class of trees with less than $m^{c}$ edges for a fixed constant $c=1.984 \ldots$

Our proof follows a recursive construction where large trees are obtained by some amalgamation process involving simpler trees. With this method, the constant $c$ could be reduced to $1.88 \ldots$ but it seems difficult to improve this value.

We conclude the paper with related open questions. 


\section{Terminology}

Our graphs are undirected and simple (with neither loops nor multiple edges). We denote by $G(V, E)$ a graph (its vertex set is $V(G)$ and its edge set is $E(G)$ (a subset of the family of all the $V(G)$-subsets of cardinality 2)). Referring to C. Thomassen [ [1, we recall some basic definitions that are useful for our purpose:

We denote by $P_{n}$ the path of size $n$.

If $x$ is a vertex then $d(x)$, the degree of $x$, is the number of edges incident to $x$.

Let $e$ be an edge of $E(G)$, the graph denoted by $G-e$ is the graph on the vertex set of $G$, whose edge set is the edge set of $G$ without $e$. We call classically this operation deletion.

Let $e=\{a, b\}$ be an edge of $G(V, E)$, we name contraction of $G$ along $e$, the graph denoted by $G / e=H\left(V^{\prime}, E^{\prime}\right)$, with $V^{\prime}=(V /\{a, b\}) \cup\{c\}$ where $c$ is a new vertex and $E^{\prime}$ the edge set which contains all the edges of the sub-graph $G_{1}$ on $V / e$ and all the edges of the form $\{c, x\}$ for $\{a, x\}$ or $\{b, x\}$ belonging to $E$.

We say that $H$ is a minor of $G$ if and only if we can obtain it from $G$ by successively deleting and /or contracting edges, in an other way, we can define the set $M(G)$ of minors of $G$ by the recursive formula :

$$
M(G)=G \cup\left(\bigcup_{e \in E(G)} M(G / e)\right) \cup\left(\bigcup_{e \in E(G)} M(G-e)\right)
$$

The notion of minor induces a partial order on graphs. We write $A \preceq B$ to mean " $A$ is a minor of $B$ ".

For technical reasons, we prefer to use the size of a tree (edge number) rather than its order (vertex number).

Finally, let us recall that, a graph $G_{\text {uni }}$ is m-universal for a sub-class $C$ of graphs if for every element $G$ of $C$ with $m$ edges, $G$ is a minor of $G_{\text {uni } i}$.

\section{A Lower Bound}

In this section, we prove that a $m$-universal tree $T_{u n i}$ for the trees has asymptotically at least $m \ln (m)$ edges. We use the fact that $T_{\text {uni }}$ has to contain all spiders of size $m$ as minors. A spider $S$ on a vertex $w$ is a tree such that $\forall v \in V(S) \backslash\{w\}, d(v) \leq 2$. We denote the spider constituted by paths of lengths $1 \leq m_{1} \leq \ldots \leq m_{k}$ by $S p\left(m_{1}, \ldots, m_{k}\right)$ (Fig.1).

Definition 1. Let $T$ be a tree, we denote by $\partial T$ the subtree of $T$ with $V(\partial T)=$ $V(T) \backslash A$, where $A$ is the set of the leaves of $T$. Also, we denote by $\partial^{k}$ the $k$-th iteration of $\partial$.

Lemma 1. $S p\left(m_{1}, \ldots, m_{k}\right) \preceq T$ involves that $\partial S p\left(m_{1}, \ldots, m_{k}\right) \preceq \partial T$. Moreover, if for all $i, m_{i}=1$ then $\partial \operatorname{Sp}\left(m_{1}, \ldots, m_{k}\right)$ is a vertex. Otherwise, put a the first value such that $m_{a}>1$, we have $\partial S p\left(m_{1}, \ldots, m_{k}\right)=S p\left(m_{a}-1, \ldots, m_{k}-1\right)$ excepted for $k=1$, in this last case we have $\partial S p\left(m_{1}\right)=S p\left(m_{1}-2\right)$. 
WG (2002)

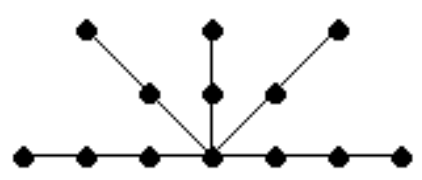

Fig.1. $S p(2,2,2,3,3)$

Proof. This just follows from an observation.

Lemma 2. For every tree $T, S p\left(m_{1}, \ldots, m_{k}\right) \preceq T \Rightarrow T$ has at least $k$ leaves.

Proof. Trivial.

Theorem 3. A m-universal tree $T_{\text {uni }}$ for the class of trees has at least $\sum_{i=1, i \neq 2}^{m}\left\lfloor\frac{m}{i}\right\rfloor$ edges.

Proof. A $m$-universal tree $T_{u n i}$ for the class of trees has to contain as minors all spiders of size $m$. So, for all $p$ it contains as minors the spiders $S p(p, \ldots, p)$ where we have $\left\lfloor\frac{m}{p}\right\rfloor$ times the letter $p$. By the lemma 1 , for all $p \leq \frac{m}{2}, S p(1, \ldots, 1) \preceq$ $\partial^{p-1} T_{u n i}$ and if $m$ is odd, $S p(1) \preceq \partial^{\left\lfloor\frac{m}{2}\right\rfloor^{-1}} T_{u n i}$. Moreover, it is clear that the terminal edges of the $\partial^{p} T_{\text {uni }}$ constitute a partition of $T_{u n i}$. By the lemma 2,

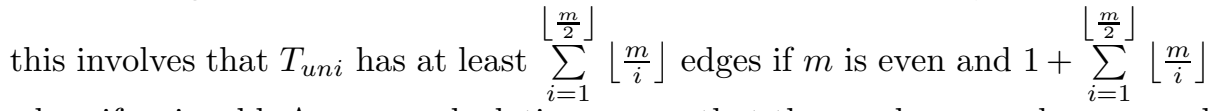
edges if $m$ is odd. An easy calculation proves that these values are always equal to $\sum_{i=1, i \neq 2}^{m}\left\lfloor\frac{m}{i}\right\rfloor$.

Proof. (of the theorem 11) it follows from the usual estimate $\sum_{i=1}^{n} \frac{1}{i} \sim \ln (n)+\gamma+$ $O\left(\frac{1}{n}\right)$ and the inequality $\sum_{i=1, i \neq 2}^{m}\left\lfloor\frac{m}{i}\right\rfloor \geq 1+\sum_{i=1, i \neq 2}^{m-1}\left(\frac{m}{i}-1\right)$.

What the above proof shows, in fact, is the following :

Corollary 1. A minimum m-universal spider for the class of spiders has $\sum_{i=1, i \neq 2}^{m}\left\lfloor\frac{m}{i}\right\rfloor$ edges.

Proof. The spider $S p\left(\left\lfloor\frac{m}{m}\right\rfloor,\left\lfloor\frac{m}{m-1}\right\rfloor, \ldots,\left\lfloor\frac{m}{2}\right\rfloor,\left\lceil\frac{m}{2}\right\rceil\right)$ is clearly a $m$-universal spider of size $\sum_{i=1, i \neq 2}^{m}\left\lfloor\frac{m}{i}\right\rfloor$ for the class of spiders, and by theorem 3 it is a minimum value. 


\section{The Main Stem}

In the sequel, we deal with rooted graph, i.e. graph $G$ where we can distinguish a special vertex denoted by $r(G)$, called the root. Conventionally, any contracted graph $G^{\prime}$ of same rooted graph $G$ will be rooted at the unique vertex which is the image of the root under the contraction mapping, we say in this case that the rooted graph $G^{\prime}$ is a rooted contraction of $G$. Note that, the contraction operator suffices to obtain all minor trees of a tree. So, we can now define the following new notion for sub-classes of rooted trees : a rooted tree $T_{\text {uni }}$ is strongly $m$-universal for a sub-classes $C$ of rooted trees if for every rooted tree $T$ in $C$ of size $m, T$ is a rooted contraction of $T_{u n i}$. The concept of root is introduced to avoid problems with graph isomorphisms that, otherwise would greatly impede our inductive proof.

For every edge $e$ of a tree $T$, the forest $T \backslash e$ has two connected components. We call $e$-branch, denoted by $B_{e}$, the connected component of $T^{\prime}$ which does not contain $r(T)$, we define the root of $B_{e}$ as $e \cap V\left(B_{e}\right)$.

A main stem of a rooted tree of size $m$ is defined as a path $P$ which is issued from the root and such that for all $e$-branches $B_{e}$ with $e \notin E(C)$, we have $\left|E\left(B_{e}\right)\right|<\left\lfloor\frac{m}{2}\right\rfloor($ Fig.2)

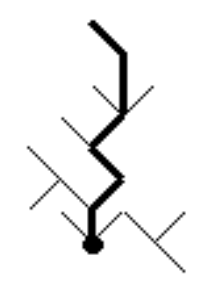

Fig.2. A main stem in bold

The following lemma suggests the procedure which will be used to find a subquadratic upper bound for universal trees. Roughly speaking, it endows every tree with some recursive structure constructed with the help of main stems.

Lemma 3. Every rooted tree has a main stem.

Proof. By induction on the size of the rooted tree. Let $T$ be a rooted tree, if $T$ has one or two edges, it is trivial. Otherwise let us consider the sub-graph $T \backslash r(T)$, which is a forest. We choose a connected component $T_{1}$ with maximum size and we denote by $b_{1}$ the unique vertex of $T_{1}$ which is adjacent to $r(T)$. Tree $T_{1}$, rooted in $b_{1}$, has, by the induction hypothesis, a main stem $B$. Then the path $\left(V(B) \cup\{r(T)\}, E(B) \cup\left\{\left\{r(T), b_{1}\right\}\right\}\right)$ is a main stem of $T$.

Remark 1. A tree may possess in general several main stems. Let us notice also that a main stem is not necessarily one of the longest paths which contain the root. 
WG (2002)

\section{The Upper Bound}

We need some new definitions. A rooted brush (Fig.3) is a rooted tree such that the vertices of degree greater than 2 are on a same path $P$ issued from the root.

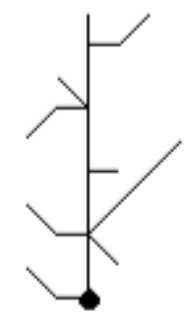

Fig.3. A rooted brush

A rooted comb $X$ (Fig.4) is a rooted brush with $d(r(X)) \leq 2$ and $\forall v \in V(X)$, $d(v) \leq 3$.

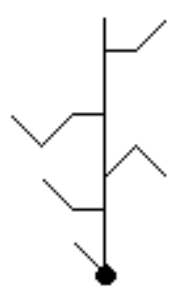

Fig.4. A rooted comb

The length of a rooted comb corresponds to the length of the longest path $P$ issued from the root which contains all vertices of degree greater than 2 .

To obtain an upper bound, we consider two building processes : the first one, a brushing $M_{B}$, maps rooted trees with a main stem into rooted brushes, the second one, a ramifying $M_{T}$, consists in obtaining a sequence of rooted trees, assuming that we have an increasing sequence of rooted combs. We note $M_{T}^{k}$ the $k$-th element of the sequence. These building processes will possess the following fundamental property:

Property 1. Let $(T, \sigma)$ a rooted tree with a main stem $\sigma$ and $\left(X_{n}\right)_{n \in \mathbb{N}}$ a sequence of rooted combs :

$$
\left(\forall T^{\prime} \preceq T, M_{B}\left(T^{\prime}, \sigma\right) \preceq X_{\left|E\left(T^{\prime}\right)\right|}\right) \Rightarrow T \preceq M_{T}^{|E(T)|}\left(\left(X_{n}\right)_{n \in \mathbb{N}}\right)
$$


Lemma 4. If building processes verify the property 1 and if for all $i$, the rooted comb $X_{i}$ is strongly $i$-universal for the class of rooted brushes then the rooted tree $M_{T}^{m}\left(\left(X_{n}\right)_{n \in \mathbb{N}}\right)$ is strongly m-universal for the class of rooted trees.

Proof. It is just an interpretation of the property. 田.

We now establish the existence of building processes which satisfy property

Brushing $M_{B}$ (Fig.5). Let $T$ be a rooted tree with a main stem $\sigma$. We are going to associate a rooted brush $B$ with it, denoted $M_{B}(T, \sigma)$ of the same size built from the same main stem $\sigma$ with the following process: every $e$-branch $B_{e}$ connected to the main stem by edge $e$ is replaced by a path of length $\left|E\left(B_{e}\right)\right|$ connected by the same edge.

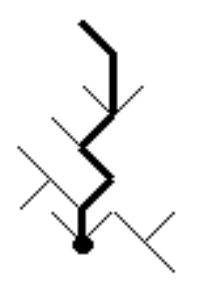

$T, \sigma$

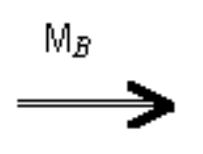

$\mathrm{M}_{B}(T, \sigma)$

Fig.5.

Ramifying $M_{T}^{k}$. For the second building process we work in two steps :

First step. Given rooted trees $T_{1}, \ldots, T_{k}$ with disjoint vertex sets, we build another rooted tree $T$, denoted $\left[T_{1}, \ldots, T_{k}\right]$, in the following way :

$$
\begin{gathered}
V(T)=\bigcup_{i=1}^{k} V\left(T_{i}\right) \cup\left\{v_{1}, \ldots, v_{k+1}\right\}, \\
E(T)=\bigcup_{i=1}^{k} E\left(T_{i}\right) \cup\left\{\left\{v_{1}, r\left(T_{1}\right)\right\}, \ldots,\left\{v_{k}, r\left(T_{k}\right)\right\}\right\} \cup\left\{\left\{v_{1}, v_{2}\right\}, \ldots,\left\{v_{k}, v_{k+1}\right\}\right\},
\end{gathered}
$$

and $r(T)=v_{1}$.

If $T_{i}=\emptyset$, conventionally $\left\{v_{i}, r\left(T_{i}\right)\right\}=\emptyset$.

Prosaically, from a path $P_{k}=\left[v_{1}, \ldots, v_{k+1}\right]$ of size $k$ and from $k$ rooted trees $T_{1}, \ldots, T_{k}$, we build a rooted tree joining a branch $T_{i}$ to the vertex $v_{i}$ of $P$ (Fig.6).

Second step. By convention, $P_{-1}=\emptyset$.

We are going to construct rooted trees $T_{k}$ in the following way :

$T_{-1}=\emptyset, T_{0}=X_{0}$, and $\forall i, 1 \leq i \leq k, T_{i}=\left[T_{\min \left(u_{1}, i-1\right)}, \ldots, T_{\min \left(u_{n_{i}}, i-1\right)}\right]$ if $X_{i}=\left[P_{u_{1}}, \ldots, P_{u_{n_{i}}}\right]$. 
WG (2002)

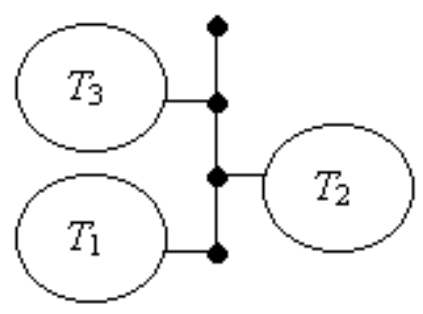

Fig.6. A rooted comb $\left[T_{1}, T_{2}, T_{3}\right]$

We can now define $M_{T}^{k}$ :

$$
M_{T}^{k}\left(\left(X_{n}\right)_{n \in \mathbb{N}}\right)=T_{k} .
$$

Lemma 5. The building processes described above verify the property 0 .

Proof. First, note that $M_{T}\left(\left(X_{n}\right)_{n \in \mathbb{N}}\right)$ is an increasing sequence. We prove the lemma by recurrence on the size $m$ of $T$. When $m=0$ or $m=1$, this is trivial. We suppose the property is verified for $T$ with size $m<m_{0}$. Let $T$ be a rooted tree of size $m_{0}$ with a stem $\sigma$, we note $e_{1}, \ldots, e_{k}$ the edges of $T$ issued from $\sigma$ which do not belong to $\sigma$. To each $e$-branch of $T$ with $e \in\left\{e_{1}, \ldots, e_{k}\right\}$ corresponds by $M_{B}$ a $e$-branch (it is a path of same size) in $M_{B}(T, \sigma)$. So there exists $k$ distinct $e$-branches $R_{1}, \ldots, R_{k}$ in $X_{m_{0}}$ that we can respectively contract to obtain each $e$-branch with $e=e_{1}, \ldots, e_{k}$ in $M_{B}(T, \sigma)$. By recurrence hypothesis, we have for $1 \leq i \leq k, B_{e_{i}} \preceq M_{T}^{\left|E\left(B_{e_{i}}\right)\right|}\left(\left(X_{n}\right)_{n \in \mathbb{N}}\right)$ and we have also $M_{T}^{\left|E\left(B_{e_{i}}\right)\right|}\left(\left(X_{n}\right)_{n \in \mathbb{N}}\right) \preceq M_{T}^{\left|E\left(R_{i}\right)\right|}\left(\left(X_{n}\right)_{n \in \mathbb{N}}\right)$. So each $e$-branch of $T$ is a minor contraction of $M_{T}^{\left|E\left(R_{i}\right)\right|}\left(\left(X_{n}\right)_{n \in \mathbb{N}}\right)$. By associativity of contraction map, we have $T \preceq M_{T}^{|E(T)|}\left(\left(X_{n}\right)_{n \in \mathbb{N}}\right)$.

In this phase, we determine a sequence of rooted combs $\left(X_{i}\right)_{i \in \mathbb{N}}$ such that the rooted combs $X_{i}$ are strongly $i$-universal for the rooted brushes.

In order to achieve this result, we define $F_{p}$ as the set of functions $f$ : $\{1, \ldots, p\} \rightarrow\left\{1, \ldots,\left\lfloor\frac{p}{2}\right\rfloor\right\}$ satisfying the following property :

$$
(\forall n \in\{1, \ldots, p\})\left(\forall i \leq\left\lfloor\frac{n}{2}\right\rfloor\right)(\exists k \in \mathbb{N})(n-i+1 \leq k \leq n \text { and } f(k) \geq i)
$$

Lemma 6. $F_{p}$ is not empty, it contains the following function $\varphi_{p}$, defined for $1 \leq i \leq p$ by :

$$
\varphi_{p}(i)=\min \left(2^{v_{2}(i)+1}-1,\left\lfloor\frac{p}{2}\right\rfloor, i-1\right)
$$

where $v_{2}(k)$ is the 2-valuation of $k$ (i.e. the greatest power of 2 dividing $k$ ).

Proof. The verification is obvious. 
Lemma 7. For every sequence $F=\left(f_{1}, f_{2}, \ldots\right)$ of functions such that $f_{i} \in F_{i}$ for $i \geq 1$ and $f_{i}(k) \leq f_{i+1}(k)$ for all $i \geq 1$ and $1 \leq k \leq i$, the rooted comb defined by $C o m b b_{m}^{F}=\left[P f_{1}^{m}, \ldots, P f_{m}^{m}\right]$ where $P f_{i}^{m}$ designs the path of size $f_{m}(m+1-i)-1$, for $1 \leq i \leq m$ is strongly $m$-universal for the rooted brushes.

Proof. By induction on $m: C o m b_{1}^{F}$ is strongly 1-universal for the rooted brushes.

Suppose that $C_{o m b}^{F}$ has all rooted brushes with $i-1$ edges as rooted contractions.

We consider two cases depending on the shape of a rooted brush $B$ of size $i$ : case 1 case 2
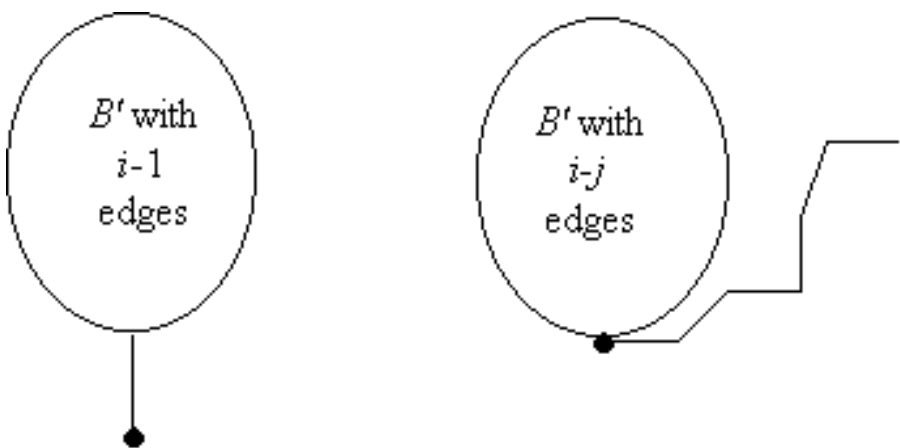

Brushes of case 1 are clearly rooted contractions of the rooted comb $\operatorname{Comb}_{i}^{F}$ $\left(B^{\prime} \preceq C o m b_{i-1}^{F}\right.$, so $\left.B \preceq\left[P_{0}, P f_{1}^{i-1}, \ldots, P f_{i-1}^{i-1}\right] \preceq C o m b_{i}^{F}\right)$. Let us study case 2 : $B^{\prime}$ is by induction hypothesis a rooted contraction of the rooted comb $C o m b_{i-j}^{F}$, moreover $C o m b_{i-j}^{F} \preceq\left[P f_{j+1}^{i}, \ldots, P f_{i}^{i}\right]$. Finally, by the property of $f_{i}$, there exists $1 \leq \alpha \leq j$, such that $P f_{\alpha}^{i}$ has more than $j$ edges. Linking these two points, we can conclude that the rooted brush $B$ is always a rooted contraction of the rooted comb $\operatorname{Comb}_{i}^{F}$.

The rooted comb built as in lemma 7 will be said to be associated to the sequence $F$ and denoted by $C o m b_{m}^{F}$.

Theorem 4. A minimum strongly $m$-universal rooted brush for the rooted brushes has $O(m \ln (m))$ edges.

Proof. Proceeding as for theorem 1, we obtain, mutatis mutandis, that a $m$ universal brush for the brushes has at least $m \ln (m)+O(m)$ edges. This order of magnitude is precisely the size of the strongly $m$-universal rooted comb $C o m b_{m}^{F}$ for the class of rooted brushes.

We have this immediate corollary : 
Corollary 2. A minimum m-universal brush for the brushes has $O(m \ln (m))$ edges.

By convention, we put $C o m b_{0}^{F}=P_{0}$ (tree reduced in a vertex)

We define $\operatorname{Tree}_{m}^{F}=M_{T}^{m}\left(\left(C o m b_{n}^{F}\right)_{n \in \mathbb{N}}\right)$.

As before, we will say that the tree built in such a way is recursively associated to the sequence $F$ and denoted by $T r e e_{m}^{F}$.

Thus, we have :

Theorem 5. The rooted tree Tree $e_{m}^{F}$ is strongly m-universal for the class of rooted trees.

We now analyze the size of $\operatorname{Tree}_{m}^{F}$.

Proposition 1. Let $F=\left(f_{1}, f_{2}, \ldots\right)$ be a sequence of functions such that $f_{i} \in F_{i}$ for $i \geq 1$. The size of a m-universal tree constructed from the sequence is given by the following recursive formula :

$$
u_{-1}=-1, u_{0}=0 \text { and } u_{k}=2 k-1+\sum_{i=1}^{k} u_{f_{k}(i)-1}
$$

Proof. It derives from the following observation :

$m$ edges constitute the main stem, we have to add $m-1$ edges to link branches to the main stem and $\sum_{i=1}^{k} u_{f_{k}(i)-1}$ edges for the branches.

Theorem 6. There is a sequence of functions $G=\left(g_{1}, g_{2}, \ldots\right)$ such that $g_{i} \in F_{i}$ and $\mid E\left(\right.$ Tree $\left._{m}^{G}\right) \mid<(2 m)^{c}$ where $c=1.984 \ldots$ is the unique positive solution of the equation $\frac{1}{2^{c}}+\frac{1}{2^{2 c}}+\frac{1}{2^{(c-1)}-1}-\frac{1}{2^{c}-1}=1$.

Proof. We take the following sequence of functions :

$g_{m}(i)=\min \left(2^{v_{2}(i)+1}, i\right)$ if $i<m$ and $i$ even, $g_{m}(i)=1$ if $i$ odd and $g_{m}(m)=$

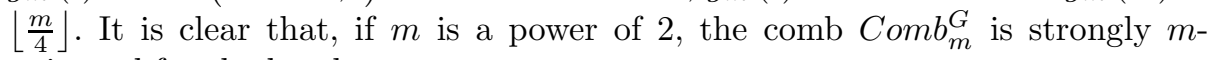
universal for the brushes.

In fact, the function $g_{m}$ takes the value $2^{v_{2}(i)+1}$ when $i$ is not a power of 2 , otherwise it is equal to $i$. Thanks to this remark and with $u_{m}<m+\sum_{i=1}^{m} u_{f_{m}(i)}$, (the sequence of sizes is increasing), we obtain $u_{2^{n}}<2^{n}+2^{n-1}+\sum_{i=2}^{n-1} 2^{n-i} u_{2^{i}}-$ $\sum_{i=2}^{n-1} u_{2^{i}}+u_{2^{n-1}}+u_{2^{n-2}}$. Thus, in evaluating the sums and reorganizing the terms, we obtain :

$$
u_{2^{n}}<\alpha_{n}+2^{n c} \beta
$$

with

$$
\alpha_{n}=2^{n-1}+1+2^{c}+\frac{1}{2^{c}-1}-\left(\frac{2^{n}}{2^{(c-1)}-1}+2^{n(c-1)}\right)
$$




$$
\beta=\frac{1}{2^{c}}+\frac{1}{2^{2 c}}+\frac{1}{2^{(c-1)}-1}-\frac{1}{2^{c}-1}
$$

Now $\alpha_{n}<0$ when $m>1$ and $\beta \leq 1$ by definition of $c$. So $u_{2^{n}}<2^{n c}$, hence $u_{m}<(2 m)^{c}$.

Remark 2. We observe that $c=\frac{\ln (x)}{\ln (2)}$, where $x$ is the positive root of $X^{4}-5 X^{3}+$ $4 X^{2}+X-2=0$.

Theorem 2 then follows since any rooted tree which is strongly $m$-universal for the rooted trees is also clearly $m$-universal for the class of trees.

\section{Conclusion and Related Questions}

When using the sequence $\Phi=\left(\varphi_{1}, \varphi_{2}, \ldots\right)$ of lemma 7 , the induction step leads to involved expressions that do not allow us to find the asymptotic behavior of the corresponding term $u_{m}$. A computer simulation gives that such a $m$-universal tree for the trees has less than $m^{1.88}$ edges. In any case, the constructive approach we proposed here, seems to be hopeless to reach the asymptotic best size of a $m$-universal tree for the trees.

Conjecture 1. The minimal size of a $m$-universal tree for the trees is $m^{1+o(1)}$.

As a possible way to prove such a conjecture, it would be interesting to obtain an explicit effective coding of a tree of size $m$ using a list of contracted edges taken in a $m$-universal tree for the trees.

A variant of our problem consists in determining a minimum tree which contains as a subtree every tree of size $m$. This is closely related to a well known still open conjecture due to Erdös and Sös (see [5]).

\section{References}

1. R. Rado, Universal graphs and universal functions, Acta Arith., 9 (1964), 331-340.

2. Z. Füredi and P. Komjàth, Nonexistence of universal graphs without some trees, Combinatorica, 17, (2) (1997), 163-171.

3. N. Robertson and P.D. Seymour, series of papers on Graph minors, Journal of combinarotics, serie B, (1983-...).

4. C. Thomassen, Embeddings and Minors, chapter 5 in Handbook of Combinatorics, ( R. Graham, M. Grötschel and L. Lovàsz, eds.), Elsevier Science B.V., 1995, 301-349.

5. P. Erdös and T. Gallai, On maximal paths and circuits of graphs, Acta Math. Sci. Hungar. 10 (1959), 337-356. 